\title{
Comment by G.G. Grabenbauer on \\ V.Vinh-Hung et al. The Effect of Adjuvant Radiotherapy on Mortality Differs According to Primary Tumor Location in Women with Node-Positive Breast Cancer in: Strahlenther Onkol 2009;185: 161-8 (No. 3) (D0I 10.1007/s00066-009-1921-z)
}

With great interest we read that the issue of radiation treatment of parasternal lymph nodes in patients with medial breast cancer is still under debate. This topic seems very interesting and should motivate radiation oncologists ...

It has been well known for decades that medial and central location of breast cancer is associated with a less favorable prognosis as compared to tumors arising in the lateral quadrants [1]. This increase in mortality risk due to medial tumor location was reported as being in the order of $15-20 \%$ [5], in patients with node-positive tumors even as high as $50 \%$ [2]. The herein reported SEER analysis by Vincent Vinh-Hung confirms this data: in women with medial tumor location following mastectomy (and one to three metastatic axillary nodes), the mortality risk increased from 1.09 (1.03-1.16) to $1.26(1.16-1.37)$ compared to women with lateral tumors (9,000 vs. 1,000 patients).

Even more interesting: recently, parasternal sentinel node detection following peritumoral injection of the radionuclide was evaluated in 855 patients. In women with positive axillary nodes on histology and positive versus negative parasternal sentinel node(s), overall survival and NED (no evidence of disease) survival differed between $91 \%$ versus $71 \%$ and $84 \%$ versus $69 \%$ [4].

Vinh-Hung et al. [3] demonstrate, that locoregional radiation treatment (treatment volume is not exactly specified, we can, however, presume that according to current clinical practice ipsilateral parasternal nodes were included with medial tumor location) improved overall survival rates in women with medial and lateral tumors. The relative reduction in mortality was $13 \%$. In the subgroup of patients with four and more positive axillary nodes and mastectomy, a marked negative impact was noted for patients with radiation treatment and medial tumor location (415 patient with irradiation, 189 without). This finding seems even more remarkable since it was not detected in the whole group of patients with medial tumors nor in any other subgroup. One can only speculate which factors may have been contributing to the negative results: high integral dose to the myocardium following radiotherapy? Larger cumulative dose of anthracyclines as compared to women with zero to three axillary nodes? Herceptin? It seems unrealistic to expect this data to be reported from the SEER analysis.

\section{What may remain as essential message?}

(1) In clinical practice, radiation exposure to cardiac structures should be kept as low as possible. This applies specifically to the left anterior descending coronary artery, which may be located in the high-dose area when treating a left-sided breast cancer.

(2) Consequently, latest techniques including intensity-modulated radiotherapy and gating should be implemented on a routine basis.

(3) The exact topographic, and imaging documentation of the location of a given breast cancer may be very desirable (preoperatively), especially because of the boost application to the tumor bed.

\section{References}

1. Grabenbauer GG. Internal mammary nodes in invasive breast cancer. To treat or not to treat? Strahlenther Onkol 2004:180:690-4.

2. Lohrisch C, Jackson J, Jones A, et al. Relationship between tumor location and relapse in 6,781 women with early invasive breast cancer. J Clin Oncol 2000;18:2828-35.

3. Vinh-Hung V, Truong P, Janni W, et al. The effect of adjuvant radiotherapy on mortality differs according to primary tumor location in women with node-positive breast cancer. Strahlenther Onkol 2009; 185:161-8.

4. Yao M, Kurland B, Smith H, et al. Internal mammary nodal chain drainage is a prognostic indicator in axillary node-positive breast cancer. Ann Surg Oncol 2007;14:2985-93.

5. Zucali R, Mariani L, Marubini E, et al. Early breast cancer: evaluation of the prognostic role of the site of the primari tumor. J Clin Oncol 1998;16:1363-6.

\section{Address for Correspondence}

Prof. Dr. Gerhard G. Grabenbauer

DiaCura-Coburg

Strahlentherapie, Radioonkologie

Ketschendorfer Straße 33

96450 Coburg

Germany

Phone (+49/9561) 2491-0, Fax 249155

e-mail:gg@diacura.de 